\title{
CLASSIFICATION OF MARKOV CHAINS WITH A GENERAL STATE SPACE
}

\author{
BY ZBYNĚK ŠIDÁK
}

\section{Communicated by J. L. Doob, September 8, 1965}

1. Introduction. Let $X$ be a general abstract space of points $x$, and $X$ a Borel $\sigma$-field of sets in $X$. Let us consider a transition function $p(\cdot, \cdot)$ of the arguments $x \in X, A \in \mathfrak{X}$ (see $[2, \mathrm{p} .190])$ which may be, however, sub-stochastic, i.e. where the usual assumption $p(x, X)=1$ is replaced by $p(x, X) \leqq 1$. The iterates $p^{(n)}$ of $p$ are defined as usual (see e.g. [2, p. 191]).

We shall always suppose that $p$ is irreducible, i.e. that the measures $\nu_{x}=\sum_{n=1}^{\infty} 2^{-n} p^{(n)}(x, \cdot)$ are equivalent for all $x \in X$. A measure $\mu$ is called sub-invariant if it is $\sigma$-finite, not identically zero, and if

$$
\int_{X} p(x, A) \mu(d x) \leqq \mu(A) \quad \text { for all } A \in \mathfrak{X} .
$$

If in (1) the sign of equality holds for all $A \in \mathfrak{X}$, then $\mu$ is called invariant.

TheOREM 1. If $\mathfrak{X}$ is generated by a denumerable class of sets, then there always exists a sub-invariant measure for any $p$.

The proof follows by a simple application of the results in [5] and [8] whenever $\sum_{n=1}^{\infty} p^{(n)}(x, A)=\infty$ for each $x$ and each $A$ satisfying $\nu_{x}(A)>0$, and by putting $\mu=\sum_{n=1}^{\infty} p^{(n)}\left(x_{0}, \cdot\right)$ whenever $\sum_{n=1}^{\infty} p^{(n)}\left(x_{0}, A\right)<\infty$ for some $x_{0}$ and some $A$ such that $\nu_{x}(A)>0$. However, there have been given also other, more complicated, conditions for the existence of a sub-invariant measure (see [8], [4]).

Let us assume in the sequel that we have some sub-invariant measure $\mu$, and that this $\mu$ is equivalent to each $\nu_{x}$. It may be seen that the latter assumption causes no loss of generality (see [8]).

Define the operator $T_{\alpha}, 1 \leqq \alpha \leqq \infty$ (see [8]), in the space $L_{\alpha}(\mu)$ by

$$
T_{\alpha} f=\int_{X} f(y) p(\cdot, d y) .
$$

2. Classification of transition functions. Our basic classification is given by the following

THEOREM 2. Each irreducible transition function $p$ having a subinvariant measure $\mu$ belongs precisely to one of the following types: either $\sum_{n=1}^{\infty} p^{(n)}(x, A)=\infty$ for each $A$ such that $\mu(A)>0$ and each $x$ ( $p$ is 
then called recurrent), or $\sum_{n=1}^{\infty} p^{(n)}(x, A)<\infty$ for each $A$ such that $\mu(A)<\infty$ and $\mu$-almost all $x$ ( $p$ is transient).

Further, each recurrent $p$ belongs precisely to one of the following types: either

$$
\lim _{n \rightarrow \infty} n^{-1} \sum_{m=1}^{n} p^{(m)}(x, A)
$$

exists and is positive for each $x$ and each $A$ such that $\mu(A)>0$ ( $p$ is called positive-recurrent), or the limit (3) is zero for each $x$ and each $A$ such that $\mu(A)<\infty$ ( $p$ is null-recurrent).

The proof is based on the individual ergodic theorem VIII.6.6. in [3] for $T_{\alpha}$, which gives the existence of (3), and on the ergodic theorem for $\sum_{m=1}^{n} T_{1}^{m} f / \sum_{m=1}^{n} T_{1}^{m} g$ in [1], which gives the rest of Theorem 2. It may be shown that this classification does not depend on the particularly chosen $\mu$ if there are more sub-invariant measures.

By the results of [4], it is easy to find that for a recurrent $p$ the sub-invariant measure $\mu$ is invariant and essentially unique.

COROLlaRY 1. If $p$ is positive-recurrent, then the measures given by (3) coincide for all $x \in X$, and are equal to a constant multiple of $\mu$; hence $\mu(X)<\infty$. If $p$ is null-recurrent, or transient and such that $p(x, X)=1$ for $\mu$-almost all $x$, then $\mu(X)=\infty$.

3. Decomposition of $T_{2}$. Let us now assume that there exists a decomposition of $X$ into $d+1$ disjoint subsets $C_{0}, C_{1}, \cdots, C_{d-1}, D$ such that $\mu(D)=0$, and $p\left(x, X-C_{j+1}\right)=0$ for each $x \in C_{j}, j=0,1, \cdots$, $d-1$ (we put here $C_{d}=C_{0}$, and in the sequel also $C_{k}=C_{d+k}$ whenever $k<0)$. Furthermore, if $A_{1}, A_{2} \subset C_{j}$ for some $j$, and $\mu\left(A_{1}\right)>0, \mu\left(A_{2}\right)>0$, let there exist, for each $x \in X$, some $n=n(x)$ such that $p^{(n)}\left(x, A_{1}\right)>0$, $p^{(n)}\left(x, A_{2}\right)>0$.

Recall also (see [10]) that a contraction operator $T$ in a Hilbert space $H$ is called completely nonunitary if the norms

$$
\|T h\|,\left\|T^{2} h\right\|, \cdots,\left\|T^{n} h\right\|, \cdots ;\left\|T^{*} h\right\|,\left\|T^{* 2} h\right\|, \cdots,\left\|T^{*_{n}} h\right\|, \cdots
$$

are not all equal to $\|h\|$, provided $\|h\| \neq 0$.

THEOREM 3. Let the mentioned assumptions be satisfied.

If $p$ is positive-recurrent, then the Hilbert space $L_{2}(\mu)$ may be decomposed into the orthogonal sum of two subspaces $L_{2}^{(u)}(\mu)$ and $L_{2}^{(0)}(\mu)$ such that the following assertions hold: $L_{2}^{(u)}(\mu)$ is the space of all functions $f$ which are constant $\mu$-almost everywhere on each $C_{j}$; both $L_{2}^{(u)}(\mu)$ and $L_{2}^{(0)}(\mu)$ reduce $T_{2}$; the part of $T_{2}$ in $L_{2}^{(u)}(\mu)$ is a unitary operator having the form 


$$
\sum_{k=0}^{d-1} e^{2 \pi i k / d} E_{k}
$$

$E_{k}$ being some projections, $E_{k} \neq 0, E_{k}^{2}=E_{k}, E_{k} E_{j}=0$ for $j \neq k, \sum_{k=0}^{d-1} E_{k}$ $=I$ (=the identical operator); the part of $T_{2}$ in $L_{2}^{(0)}(\mu)$ is a completely nonunitary contraction.

If $p$ is null-recurrent or transient, then $T_{2}$ itself is a completely nonunitary contraction.

The proof is based on the theorem of [10] and on the following two auxiliary assertions: If there exists a function $f \in L_{2}(\mu)$ such that $f \not \equiv 0$ and $\left\|T_{2}^{n} f\right\|=\|f\|$ for all $n=1,2, \cdots$, then $p$ is positive-recurrent. On the other hand, if $p$ is positive-recurrent, and $f \in L_{2}(\mu)$, then $\left\|T_{2}^{n} f\right\|=\|f\|=\left\|T_{2}^{*} n f\right\|$ for all $n=1,2, \cdots$ if, and only if, $f$ is constant $\mu$-almost everywhere on each $C_{j}$.

Corollary 2. Suppose that $p$ is positive-recurrent, $r$ is one of the numbers $0,1, \cdots, d-1$, and $A \subset C_{j}$. Then $p^{(m d+r)}(x, A)$ converges weakly in $L_{2}(\mu)$, for $m \rightarrow \infty$, to the function

$$
\begin{aligned}
p_{A}^{(r)}(x) & =d \mu(A)[\mu(X)]^{-1} & & \text { for } x \in C_{j-r}, \\
& =0 & & \text { for } x \in C_{k}, k \neq j-r, \\
& =\text { arbitrary } & & \text { for } x \in D .
\end{aligned}
$$

Furthermore, if $B \subset C_{k}$, then there is a complex-valued function $\phi_{A, B}$ integrable over $[0,2 \pi]$ such that, for all $m=1,2, \cdots$ and for $k=j-r$,

$$
\int_{B} p^{(m d+r)}(x, A) \mu(d x)=d \mu(A) \mu(B)[\mu(X)]^{-1}+\int_{0}^{2 \pi} e^{i m d t} \phi_{A, B}(t) d t .
$$

If $p$ is null-recurrent or transient, and if $\mu(A)<\infty$, then $p^{(n)}(x, A)$ converge weakly in $L_{2}(\mu)$ to 0 , for $n \rightarrow \infty$. Furthermore, if also $\mu(B)<\infty$, then there is a complex-valued function $\phi_{A, B}$ integrable over $[0,2 \pi]$ such that, for all $n=1,2, \cdots$,

$$
\int_{B} p^{(n)}(x, A) \mu(d x)=\int_{0}^{2 \pi} e^{i n t} \phi_{A, B}(t) d t .
$$

This corollary clearly embraces the classical results on the convergence of transition probabilities in denumerable Markov chains, as well as their strengthening expressed by integral representations of transition probabilities in [6], [7]. It also strengthens some theorems in [9] for a general $X$.

Full proofs of the results announced here, together with a number of related results, will be published later in the Transactions of the 
Fourth Prague Conference on Information Theory, Statistical Decision Functions, Random Processes.

\section{REFERENCES}

1. R. V. Chacon and D. S. Ornstein, A general ergodic theorem, Illinois J. Math. 4 (1960), 153-160.

2. J. L. Doob, Stochastic processes, Wiley, New York, 1953.

3. N. Dunford and J. T. Schwartz, Linear operators. Vol. I, Interscience, New York, 1958.

4. J. Feldman, Subinvariant measures for Markoff operators, Duke Math. J. 29 (1962), 71-98.

5. T. E. Harris, The existence of stationary measures for certain Markov processes, Proc. Third Berkeley Symposium, Vol. II, pp. 113-124. Univ. of California Press, Berkeley, Calif., 1956.

6. D. G. Kendall, Integral representations for Markov transition probabilities, Bull. Amer. Math. Soc. 64 (1958), 358-362.

7. - Unitary dilations of Markov transition operators, and the corresponding integral representations for transition-probability matrices, Probability \& Statistics, The Harald Cramér Volume, Wiley, New York, 1959, pp. 139-161.

8. E. Nelson, The adjoint Markoff process, Duke Math. J. 25 (1958), 671-690.

9. Z. Sidák, Integral representations for transition probabilities of Markov chains with a general state space, Czechoslavak. Math. J. 12 (87) (1962), 492-522.

10. B. Sz.-Nagy and C. Foiaş, Sur les contractions de l'espace de Hilbert. IV, Acta Sci. Math. (Szeged) 21 (1960), 251-259.

Mathematical Institute, Czechoslovak Academy of Sciences 